\title{
Der Prüfungsmix an der Medizinischen Universität Wien
}

\author{
Monika Himmelbauer (iD) • Desiree Koller · Andjela Bäwert · Werner Horn
}

Eingegangen: 30. März 2018 / Angenommen: 7. September 2018 / Online publiziert: 28. September 2018

(C) Der/die Autor(en) 2018

\begin{abstract}
Zusammenfassung Die vorliegende Arbeit bietet einen Überblick über den bestehenden Prüfungsmix für das Studium der Humanmedizin an der Medizinischen Universität Wien. Es werden die Einsatzgebiete von speziellen Prüfungsformaten sowie die Vor- und Nachteile der einzelnen Prüfungsformen beschrieben. Dargestellt werden summative schriftliche Prüfungen, Assessment von praktischen Skills und Fertigkeiten (OSCE), mündlich-praktische Prüfungen sowie formative Prüfungs- und Überprüfungsarten. Studien zeigen, dass wiederholtes Prüfen $\mathrm{zu}$ einem höheren Lernzuwachs führt. Es stellt sich daher die Herausforderung, geeignete Methoden zur kontinuierlichen Beurteilung des Lernfortschritts im Studium zu entwickeln und entsprechend anzuwenden. So sollten neben den schriftlichen summativen Prüfungen vermehrt mündliche und praktische Prüfungsformen im Curriculum eingesetzt werden.
\end{abstract}

Schlüsselwörter Assessment · Prüfungsmethoden · Prüfungsformate

\section{The examination mix at the Medical University Vienna}

Summary The present work describes a curriculum design with the instrument of an examination mix for the study of medicine at the medical University of Vienna. The fields of application of special examination formats as well as the advantages and disadvantages

\footnotetext{
M. Himmelbauer $(\varangle) \cdot$ D. Koller · A. Bäwert · W. Horn Teaching Center, Medical University Vienna, Spitalgasse 23, 1090 Vienna, Österreich monika.himmelbauer@meduniwien.ac.at; desiree.koller@meduniwien.ac.at; andjela.baewert@meduniwien.ac.at; werner.horn@meduniwien.ac.at
}

of the individual examination forms are presented. Types of summative written examinations, assessment of practical skills and abilities (OSCE), oral-practical examinations, as well as formative examinations are illustrated. Studies show that repeated testing leads to higher learning gains. Therefore, the challenge is to develop suitable methods for the continuous assessment of the learning progress in the study and to apply accordingly. Thus, in addition to the written summative exams, more and more oral and practical forms of examinations in courses with continuous assessment character should be used.

Keywords Assessment · Test methods · Test formats

\section{Einleitung}

Untersuchungen zufolge richten Studierende ihr Lernen darauf aus, was sie vermuten, dass geprüft wird und in welcher Form geprüft wird (vgl. [1-4]). So haben z. B. die Gewichtung eines Faches im Rahmen einer Gesamtprüfung, das Prüfungsformat in Beziehung zur Dimension von Kompetenzen (siehe Abb. 1) sowie die Konsequenzen des „Nicht-Bestehens“ einer Prüfung Einfluss auf die Motivation der Studierenden zu lernen. In diesem Sinne kann die Relation von Lehre/ Lernen/Prüfen als „the assessment drives learning“ verstanden werden [4, 5]. Eine Anpassung zwischen dem, was gelehrt wird, dem was die Studierenden lernen und dem, was schlussendlich geprüft wird, ist daher eine wichtige Voraussetzung für ein qualitätsvolles Curriculum.

Traditionell eingesetzte Prüfungsformate wie schriftliche und mündliche Prüfungen können den Dimensionen von ärztlichen Kompetenzen nur teilweise gerecht werden. Aus diesem Grund spielt das Assessment im Bereich der medizinischen Aus- und 


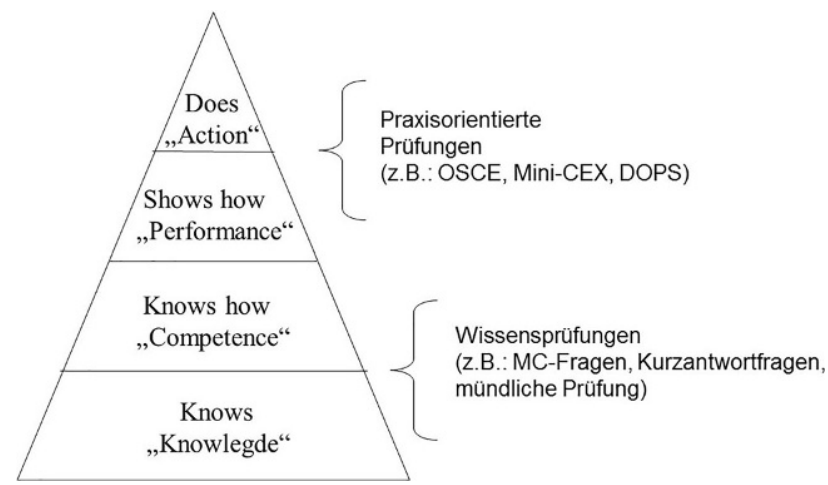

Abb. 1 Dimensionen von Kompetenzen aus Miller's Framework of Clinical Assessment (@ Miller, 1990 [7])

Weiterbildung sowie in der Lehr- und Lernforschung eine besondere Rolle [6].

Im internationalen Vergleich kommen praktischen Prüfungen im Medizinstudium immer mehr Bedeutung zu. So demonstriert eine Studie aus Großbritannien an 23 Medical Schools, dass sich AbsolventInnen von Studiengängen mit hohem Anteil an praktischen Prüfungen besser auf die Praxis als Arzt/Ärztin vorbereitet fühlen als AbsolventInnen von Studiengängen mit einem hohen Anteil an schriftlichen Prüfungen [8].

Eine große Herausforderung für das Prüfungswesen an der Medizinischen Universität Wien (MedUni Wien) sind die hohen Studierendenzahlen von ca. 660 Studierenden pro Jahrgang. Ein wesentlicher Punkt bei der Gestaltung des Prüfungswesens ist, für diese Studierendenzahlen geeignete Prüfungsformate und valide Prüfungsformen zu finden. Die vorliegende Arbeit soll einen Überblick über den bestehenden Prüfungsmix, die Vor- und Nachteile der einzelnen Prüfungsformen sowie die Einsatzgebiete von speziellen Prüfungsformaten geben. Zudem soll ersichtlich werden, in welchen Bereichen zusätzliche Assessmentinstrumente notwendig wären.

Das Medizinstudium an der MedUni Wien ist als integriertes Curriculum aufgebaut. Es stehen nicht mehr die Fächer als einzelne im Vordergrund, sondern ein integrierter Fächerkanon (häufig organzentriert; siehe Abb. 2) sowie eine Vernetzung von Vorklinik und Klinik. Im Sinne der Angleichung von Lehre/Lernen/ Prüfen ist daher auch ein Großteil des Prüfungssystems integriert gestaltet.

\section{Summative Prüfungen - Multiple Choice und Kurzantwortfragen}

\section{Summative Integrierte Prüfungen zur Feststellung des theoretischen Wissens}

Um kompetenzbasierte Ausbildungsstandards zu garantieren, werden u. a. summative Prüfungen (Abb. 3) eingesetzt. Die Ergebnisse geben Aufschluss, ob ein gefordertes Kompetenzniveau erreicht wurde. Die se- lektive Funktion dieser Prüfung wirkt sich auf den Studienfortschritt aus [9].

Die summativen integrierten Prüfungen finden am Ende eines Semesters (SIP1a, SIP1b und SIP4a) oder Studienjahres (SIP2, SIP3 und SIP5a) statt (siehe roter Balken in Abb. 2). Sie beinhalten im ersten und zweiten Studienabschnitt eine integrative Prüfung über die Inhalte der Blöcke und im dritten Studienabschnitt über die Inhalte der Tertiale [10].

Für die große Studierendenkohorte bieten sich Multiple-Choice-Fragen als effizientes Prüfungsformat an. An der MedUni Wien werden die zwei gängigen Fragetypen Multiple-Choice-One-Best-Answer ${ }^{1}-$ und -Pick- $\mathrm{N}^{2}$ mit jeweils fünf Antwortmöglichkeiten nebeneinander verwendet. Die Beantwortung beschränkt sich bei den Multiple-Choice (MC)-Fragen auf ein Ausmalen eines oder mehrerer Kästchen am Antwortbogen. Die Auswertung erfolgt automatisiert.

Die MC-Prüfung evaluiert primär den Umfang und das Vorhandensein von Fachwissen und kann nur in begrenztem Ausmaß die Fähigkeit des klinischen Denkens prüfen. Sie ist eine objektive, transparente und bei großen KandidatInnenzahlen ökonomische Prüfungsform. Die hohe Fragenanzahl pro Prüfung erlaubt eine repräsentative Inhaltsstichprobe. Somit können zuverlässige Ergebnisse und inhaltlich gültige Prüfungen erreicht werden. Dem Erstellungsaufwand der Prüfung, dem ein umfassender Fragenentwicklungs-Prozess durch geschulte FragenautorInnen und ein Pre-Review-Prozess durch Komitees, die sich aus unterschiedlichen Fachexperten zusammensetzen, vorausgeht, folgt eine umfassende Qualitätssicherung der Fragen in Form eines Post-Review-Prozesses. Der Fragenerstellung und Prüfungszusammenstellung liegt ein Blueprint zugrunde. Die statistischen Fragen- und Prüfungsanalysen ermöglichen eine stetige Optimierung des Fragenpools. Bei kleineren KandidatInnenzahlen $(n<50)$ können die statistischen Informationen allerdings nur bedingt genutzt werden.

Das Formulieren formal und sprachlich korrekter, anwendungsorientierter MC-Fragen, mit eindeutigen, bestmöglichen Antworten, guten Distraktoren (falsche Antwortalternativen) und ohne ungewollte Lösungshinweise (Cues), ist jedoch eine anspruchsvolle Aufgabe. Cues sind zu vermeiden, damit die Prüfung reliabel bleibt. Die beiden MC-Fragetypen verleiten zum Erstellen reiner Faktenwissensfragen, die bei Prüfungen im ersten und teils auch im zweiten Studienjahr zum Festigen und Prüfen von Grundlagenwissen berechtigte Anwendung finden.

Alternativ werden Prüfungen im Kurzantwort-Fragen-Format ${ }^{3}$ angeboten. Es handelt sich dabei um

\footnotetext{
1 Fragen mit einer bestmöglichen Antwort.

2 Fragen mit zwei bis drei bestmöglichen Antworten.

3 Fragen mit maximal drei richtigen Antworten. Die erwartete Antwort kann ein Wort, eine Sinneinheit, ein kurzer Satz, eine Ergänzung einer Zeichnung oder eine Reihung eines Ablaufes sein.
} 


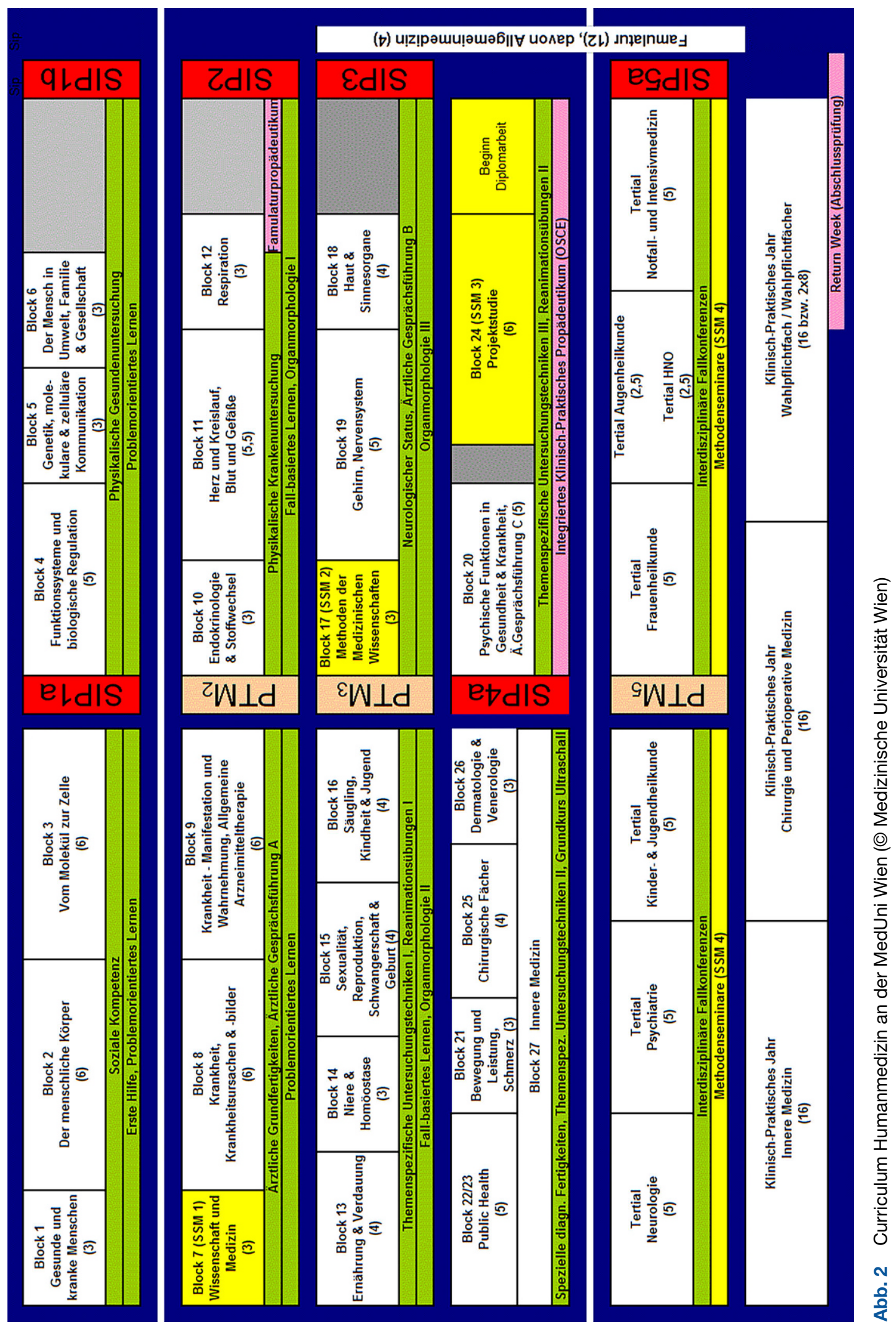




\section{Lehre}

Lernen

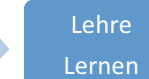

Lernen

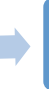

Lehre

Lernen summative Prüfung
Abb. 3 Summative Prüfung

Fragestellungen mit einem Schlüssel an richtigen Antworten zum standardisierten Korrigieren durch Block-/TertialkoodinatorInnen. Der Vorteil dieser offenen Antwortform ist, dass die KandidatInnen ihr Wissen aktiv formulieren können, im Vergleich zum passiven Wiedererkennen einer äquivalenten MCFrage. Dieser Fragetyp lässt in der Regel eine anspruchsvolle Beantwortung zu. Zudem ermöglicht dieser Typ das Prüfen von Anwendungswissen in Form von Verstehen, Interpretieren und Anwenden des vorhandenen Faktenwissens. Der Nachteil liegt allerdings im Aufwand an Auswertungsarbeit bei einer großen Anzahl an KandidatInnen [11].

Der Einsatz von Multiple-Choice-Fragen und Kurzantwortfragen ist im Sinne der Kompetenzstufen nach Miller [7] der ersten Stufe „Knows“ und zweiten Stufe „Knows how“ zuzuordnen (siehe Abb. 1).

\section{Lehrveranstaltungsprüfungen}

Im Zuge des Studiums absolvieren die Studierenden zwei Lehrveranstaltungsprüfungen über die Pflichtlehrveranstaltungen der speziellen Studienmodule (SSM), die die Grundlagen der wissenschaftlichen Ausbildung vermitteln: SSM 1 „Wissenschaft und Medizin“ und SSM 2 „Methoden der Medizinischen Wissenschaften“ [10]. Im Rahmen dieser SSMs eignen sich die Studierenden grundlegende Kenntnisse wissenschaftlichen Arbeitens in Hinblick auf die Vorbereitung ihrer Diplomarbeit an. Für die Lehrveranstaltungsprüfungen kommen sowohl Multiple-Choiceals auch Kurzantwortfragen-Prüfungen zum Einsatz.

\section{Prüfungsvielfalt in Lehrveranstaltungen mit im- manentem Prüfungscharakter}

Zur Leistungsfeststellung in Lines, Seminaren, Praktika und Wahlpflichtfächern (siehe Abb. 2) dienen unterschiedliche Prüfungsformate, die die erreichten Fertigkeiten und Fähigkeiten der Studierenden überprüfen. Beispiele der verwendeten Prüfungsformate, die in Lehrveranstaltungen mit immanentem Prüfungscharakter zur Anwendung kommen, sind in Tab. 1 dargestellt. Anschließend sind einige dieser Prüfungsformen genauer erklärt.

Zur Unterstützung der Durchführung von OnlinePrüfungen wird die Lernplattform Moodle ${ }^{4}$ verwendet. Die Studierenden können dabei durch Zugriff via Internet in einem definierten Zeitfenster Ihre Prüfung(sfragen) bearbeiten. Es steht eine Reihe von Fragenformaten zur Verfügung (z.B. MC- und Kurz-

\footnotetext{
${ }^{4}$ https://moodle.meduniwien.ac.at.
}

antwortfragen, Ergänzungsfragen, Zuordnungsfragen, Bildfragen mit Markierungs-Option).

Anhand von Key-Feature-Fällen üben Studierende das Treffen „kritischer“ Entscheidungen, um klinische Probleme lösen zu lernen. Der Einsatz von Key-Feature-Fällen hat das Ziel klinisch-diagnostisches Denken zu fördern. Diese Fälle bestehen aus der Darstellung einer klinischen Situation, gefolgt von drei bis fünf Sub-Fragen, die das Erkennen der „Key-Features“ des Falles zum Ziel haben. Diese sind entweder als MC-, Long-Menu oder Kurzantwortfragen formuliert [12].

Mittels Video wird im Kommunikationsunterricht ein simuliertes Gespräch zwischen einem Studierenden (Arzt/Ärztin) und einem Simulierten Patienten (SP) aufgezeichnet. Positive und negative Aspekte des Gesprächs sollen vom betreffenden Studierenden mit Hilfe des Videoreviews sowie des Feedbacks der GruppenteilnehmerInnen reflektiert werden.

Im Rahmen einer Strukturierten Beobachtung helfen Checklisten den Lehrenden/Prüfenden das konkrete Verhalten eines Studierenden (z.B.: bei der Reanimation einer Puppe) zu dokumentieren, um möglichste objektiv Rückmeldung über die Leistung des Studierenden geben zu können.

\section{Gesamtprüfungen von praktischen Skills und Fer- tigkeiten (OSCE)}

Unter einer „Objective Structured Clinical Examination" OSCE wird ein Methodenmix innerhalb eines flexibel gestaltbaren, klinisch-praktischen Prüfungsrahmens verstanden [13]. Ausgehend von den im Curriculum vorangegangenen Lehrveranstaltungen und Praktika wird am Ende des 2. Studienjahres, im Famulaturpropädeutikum (FamProp), und am Ende des 4. Studienjahres, im Integrierten Klinischen PflichtPraktikum (IKPP), eine Abschluss-Kompetenz-Überprüfung durchgeführt. Dafür werden mehrere unterschiedliche Prüfungsstationen konstruiert, an denen klinische Fertig- und Fähigkeiten überprüft werden. Jedes klinische und vorklinische Fach kann prinzipiell bei einer OSCE vertreten sein. Derzeit bewerben sich jedes Jahr ungefähr 13-15 unterschiedliche Kliniken und Departments für eine OSCE-Station.

Die Studierenden bestreiten bei diesem Prüfungsformat einen genau vorgegeben Parcours, wobei sie entweder einzeln, wie beim IKPP, oder als Prüfungspaar, so wie es beim FamProp der Fall ist, von einer Station zu nächsten gehen. Jene Studierenden, die paarweise die OSCE durchlaufen, stellen sich gegenseitig als Assistenz ärztlicher Tätigkeiten oder als PatientInnen zur Verfügung. Bei jeder Station beobachtet ein/eine Lehrende/r die ärztlichen Handlungsschritte der Studierenden und hält diese in einer vorgegebenen, für die jeweilige Station einheitlichen, Checkliste fest. Die Aufgabenstellungen reichen hierbei vom Vorzeigen der Reanimation an Erwachsenen- bzw. Kinder-Modellen über die Durchführung eines Arzt/ 
Tab. 1 Beispiele für die wichtigsten Prüfungsformate in Lehrveranstaltungen mit immanentem Prüfungscharakter

\begin{tabular}{|l|l|}
\hline Prüfungsformat & Lehrveranstaltung (siehe Abb. 2) \\
\hline (Strukturierte), mündliche Prüfung & Block 2, Block 9 \\
\hline Key-Feature-Fälle (via Moodle) & Interdisziplinäre Fallkonferenzen \\
\hline Seminar- bzw. Praktikumsarbeiten & SSM 3 \\
\hline Referate/Norträge & SSM 1-3, Problem-orientiertes Lernen \\
\hline Präsentation von Fällen & Return Week \\
\hline Praktische Demonstrationen & Organmorphologie I-III \\
\hline Videoreviews & Ärztliche Gesprächsführung A \\
\hline Strukturierte Beobachtung & Erste-Hilfe-Praktikum \\
\hline
\end{tabular}

ÄrztInnen-PatientInnen-Gesprächs mit SimulationspatientInnen, der Interpretation von Röntgenbildern, Laborbefunden sowie von EKGs mit nachfolgender Diagnosestellung bis hin zur Vorführung der gynäkologischen Untersuchung am Modell. Die Bewertung erfolgt auf Basis der, in den standardisierten Checklisten, notierten Beobachtungen, für die anhand eines vorher festgesetzten Notenschlüssels Punkte für die jeweilige Station vergeben werden. Die Studierenden haben für jede Station einen vorgegebenen Zeitrahmen, in dem sie die Aufgabe bewältigen müssen. Nach Verstreichen der Zeit erfolgt ein akustisches Signal als Hinweis, dass der Wechsel zur nächsten Station durchgeführt werden soll.

Solchermaßen gestaltete Prüfungen ermöglichen es, praktische Aspekte klinischer Kompetenz (siehe Abb. 1, Stufe „Shows how“) zu erfassen, wobei das Abprüfen von reinem Faktenwissen in den Hintergrund rückt. Die Überprüfung von Handlungskompetenzen und klinisch-praktischen Untersuchungstechniken stellt eine essentielle Ergänzung zu reinen Wissensüberprüfungen dar. Der interdisziplinäre Charakter dieses Prüfungsformats fügt sich gut in das integrierte Curriculum ein. Hierbei hat die Standardisierung der Prüfungssituation für jeden der ca. 660 PrüfungskandidatInnen eines Studienjahres hohe Priorität. Die Rahmenbedingungen und die Abläufe an den Prüfungsstationen sind standardisiert vorgegeben, sodass eine ausreichende Objektivität gewährleistet werden kann.

\section{e-OSCE}

Bereits vor einigen Jahren wurde begonnen, das Papier und Bleistift-Format der OSCE durch eine elektronisch gestützte Prüfung am Tablet zu ersetzen. Hierzu wurde eine spezielle Prüfungssoftware mit den Anforderungen der OSCE verknüpft. Die „pen and paper“-Beurteilungen stehen als Reservemöglichkeit bei Serverproblemen zur Verfügung. Die Akzeptanz unter den Lehrenden nach Einführung dieses Prüfungsformates war sehr hoch, da die elektronische Form der Administration bei einer praktischen Prüfung zu einer Standardisierung der Dokumentation führte. Fehler und Ungenauigkeiten wurden im Vergleich zur Papierund Bleistift-Dokumentation stark minimiert [14].

\section{Mündlich-praktische Prüfung in den Tertialen des 5. Studienjahres}

\section{Tertialüberprüfung}

Diese mündlich-praktische Tertialüberprüfung wird im Rahmen einer visitenartigen Situation anhand eines/r Patienten/in durchgeführt (siehe Abb. 1, Stufe „Does“). Sie entspricht im Format der Mini-Clinical Evaluation Excercise (Mini-CEX) [15]. Die Beurteilung erfolgt durch Beobachtung des Studierenden über den gesamten Prüfungszeitraum. Es werden verschiedene Bereiche bewertet: Anamnese, Klinische Untersuchung, Kommunikative Fähigkeiten und Professionelles Verhalten. Die Beurteilung der Durchführung der gestellten Aufgabe erfolgt nach einer vorgegebenen Skala und muss auf einem Bogen zur Bewertung der klinisch-praktischen Fertigkeiten dokumentiert werden. Anschließend erfolgt ein Feedback durch den/die Prüfer/in. Die Dauer beträgt ca. $20 \mathrm{~min}$ (15 min Assessment, 5 min Feedback).

Aus Gründen der Ökonomie werden aus den sieben Tertialen des 5 . Studienjahres (siehe Abb. 2) für jeden Studierenden zwei Tertiale gelost, in denen diese geprüft werden. Prüfungsinhalt sind grundsätzlich die Lernziele des Logbuchs und die im Tertial erworbenen Fertigkeiten und Fähigkeiten. Die zugrundeliegenden theoretischen Kenntnisse bilden eine Voraussetzung, um klinische Kompetenzen demonstrieren zu können. Eine Überprüfung des darüber hinausgehenden fachspezifischen Wissens steht nicht im Fokus der Tertialüberprüfung. Das Instrument zielt insbesondere darauf ab, Feedback für die Studierenden zu ihren praktisch-klinischen Kompetenzen zu liefern.

Die Ergebnisse können subjektiv sein, wenn die PrüferInnen nicht trainiert sind. Ein entsprechendes Ratertraining ist daher vorteilhaft. Um ein einheitliches Beurteilungsniveau zu erreichen, gibt es eine genaue schriftliche Anleitung für die PrüferInnen, welche Aspekte zu beurteilen sind. Die Reliabilität und Validität sind abhängig von der Tatsache, dass die Studierenden in verschiedenen Realsituationen (unter- 
schiedliche PatientInnen in unterschiedlichen klinischen Settings) beobachtet werden, und sind daher von Fall zu Fall unterschiedlich hoch. Ein Vorteil des Mini-CEX liegt in der raschen Durchführung und unmittelbaren Beurteilung inklusive Feedback.

\section{Logbuch zur Überprüfung der erreichten Lernziele}

Zur Dokumentation des Lernfortschritts sind Studierende aufgefordert ein Logbuch über die in den Tertialen des 5. Studienjahres geforderten Lernziele zu führen. Dies dient dem Nachweis der erlernten praktischklinischen Fertigkeiten in den sieben Tertialen. Die im Logbuch enthaltenen Lernziele müssen entsprechend ausgefüllt und von den Lehrenden bestätigt werden. Im klinischen Abschnitt des Studiums kommt dem Erwerb von klinischen Fertigkeiten eine besondere Bedeutung zu. Dementsprechend müssen auch vorgegebene Kompetenzniveaus erreicht werden. Diese sind im Logbuch [16] festgelegt als:

1. Theoretische Kenntnis (Lerninhalt gehört haben)

2. Praktische Erfahrung (Lerninhalt gesehen und ev. einige Male selbst durchgeführt haben)

3. Praxissicherheit (sicheres Beherrschen des Lerninhalts, Problemlösung).

\section{Begleitende und abschließende Beurteilung im klinisch-praktischen Jahr (KPJ)}

Im 48 Wochen dauernden KPJ im 6. Studienjahr erfolgt eine kontinuierliche Überprüfung von klinischpraktischen Fertigkeiten und Fähigkeiten anhand von PatientInnen im Rahmen der Ausbildung in den Lehrkrankenhäusern. Begleitend müssen die Studierenden ein Logbuch und Portfolio führen, in dem die erreichten Lernziele und die durchgeführten Aufgabenstellungen dokumentiert werden. Eine Abschlussprüfung findet nach Absolvierung des KPJ statt (Return-Week).

Alle 14 Tage ist über den gesamten Zeitraum des KPJ als ausgewiesene Prüfungsleistung ein Mini-CEX (Mini-Clinical Evaluation Exercise) oder DOPS (Directly Observed Procedural Skills) durchzuführen. Diese Überprüfung ist in den klinischen Alltag integriert (siehe Abb. 1, Stufe „Does“). Sie wird zwischen MentorIn und Studierenden vorab vereinbart und analog der Tertialüberprüfung auf einem Beurteilungsblatt dokumentiert.

Der DOPS ist eine klinisch-praktische Prüfung von Skills mit Feedback, die wie die oben beschriebene Mini-CEX abgewickelt wird (z. B. Chirurgische Desinfektion des OP-Gebietes vor Operation im KPJ-Tertial „Chirurgie und Perioperative Medizin“).

Auch hier ist das Feedback die wesentliche Stärke der Beurteilung: „Das Feedback zur demonstrierten Leistung ist von zentraler Bedeutung. Denn nur durch Rückmeldung zu ihrem Entwicklungsbedarf können die Studierenden ihre klinischen Fertigkeiten und Fähigkeiten kontinuierlich verbessern. Das Feedback ermöglicht den Studierenden, ihren Lernfortschritt in
Relation zu den Lernzielen zu erkennen. Teil des Feedbackgespräches ist auch die Erarbeitung von klaren, überprüfbaren Zielen und Handlungen zur Verbesserung der Schwächen gemeinsam mit der/dem Studierenden. Die Formulierung eines Handlungsplans ist der wichtigste Schritt für den Erfolg der begleitenden Beurteilung“" [17].

\section{Return-Week}

Nach Absolvierung der KPJ-Tertiale kehren Studierende an die MedUni Wien zurück, um in der ReturnWeek eine abschließende Prüfung der im KPJ erworbenen klinischen Kompetenzen zu absolvieren. Dabei werden von den Studierenden Fallpräsentationen aus dem KPJ vorgestellt und in der Gruppe gemeinsam mit einem/r klinischen Fachexperten/in diskutiert. Anschließend erfolgt ein mündliches Assessment zu den in den Pflichttertialen „Innere Medizin“ sowie „Chirurgie und Perioperative Medizin“ (siehe Abb. 2) durchgeführten Aufgabenstellungen.

\section{Nachweis wissenschaftlicher Kompetenz (Di- plomarbeit und Diplomprüfung)}

Die Diplomarbeit ist eine schriftliche Arbeit, die einem wissenschaftlichen Fachgebiet der Medizin zugeordnet ist und mind. 50 Seiten umfassen soll. Das Ziel der Diplomarbeit ist es, die Studierenden mit erforderlichen wissenschaftlichen Kompetenzen auszustatten, die sie für den Beruf als Arzt bzw. Ärztin benötigen. Daher ist es besonders wichtig, dass das Thema eigenständig erarbeitet wird und den Standards einer wissenschaftlichen Arbeit entspricht. Voraussetzungen für die Anfertigung einer Diplomarbeit sind neben der Absolvierung von SSM 1 und SSM 2 - eine Projektstudie zur Planung der Diplomarbeit (SSM 3) und die Absolvierung von zwei Methodenseminaren (SSM 4), die Unterstützung zur Fertigstellung der Diplomarbeit bieten.

Nach Approbation der Diplomarbeit ist diese im Rahmen einer mündlich-kommissionellen Diplomprüfung, die dem wissenschaftlichen Fachgebiet zugeordnet ist, zu verteidigen (Defensio).

Formative Prüfungs- und Überprüfungsarten als Chance für den Aufbau des Reflexionsvermögens und für die Optimierung des Lernverhaltens

\section{Formative Integrierte Prüfungen zur Selbstüberprüfung des theoretischen Wissens}

Prüfungen, die während eines Lehr- und Lernprozesses an die Studierenden Feedback über ihre Leistung und ihren Wissensfortschritt und Rückmeldungen an die Lehrenden über die Stärken und Schwächen ihres Unterrichts anbieten, werden als formative Prüfungen bezeichnet (Abb. 4). Die Ergebnisse vergleichen, ähnlich den summativen Prüfungen, den Ist- und Soll- 


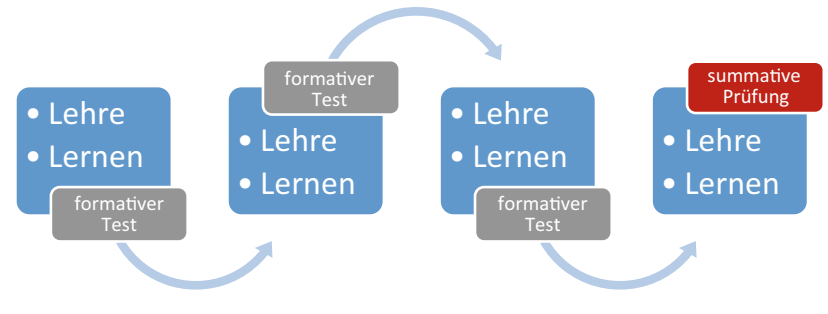

Abb. 4 Formative Prüfung

Stand der Leistung, nur ohne Konsequenz der Selektion [9]. Die Benotung erfolgt in der Regel „mit Erfolg teilgenommen“ und gilt als Voraussetzung für die Absolvierung der SIP [10].

Formatives Prüfen ist ein wichtiger Bestandteil, um vertieftes Lernen zu ermöglichen [18]. Der Prozess der Optimierung des Lernverhaltens beginnt mit dem Aufdecken der Wissenslücken und geht über die verstärkte Auseinandersetzung mit der Materie bis hin zum Verstehen und Interpretieren des Gelernten. Studierende sollen in ihrem Lernprozess unterstützt, motiviert und zu selbstgesteuertem Lernen angeregt werden. Dafür ist es notwendig, Studierenden geeignetes Feedback zu geben, um ihre Selbstreflexion zu aktivieren. Dies wiederum führt zu einer Leistungssteigerung bei summativen Prüfungen [19]. Studien belegen den positiven Effekt von formativem Feedback. Es regt kontinuierliches Lernen an und ermöglicht einen soliden Aufbau der Wissensbasis [20]. Ferner gewinnt das "assessment for learning“ (z. B.: FIP), im Vergleich zum „assessment of learning“ (SIP), „als zentrale Aufgabe einer anspruchsvollen inhaltlichen und formalen Gestaltung eines Gesamtprüfungsprogramms“, zunehmend an Bedeutung [21]. Die Idee des „assessment for learning“ ist nicht neu. Martinez und Lipson hatten diesen Vorschlag, dass Lehren und Prüfen einander ergänzen und stärken können, bereits 1989. Sie beschreiben ein „Assessment for Learners" und ein "Assessment for Teachers“ [22, 23].

Die formativen integrierten Prüfungen werden an der MedUni Wien mittels Progress Test Medizin (PTM) im zweiten, dritten und fünften Studienjahr durchgeführt.

\section{Progress Test Medizin (PTM) als spezielle Prüfungsart}

Das Konzept des Progress Test Medizin (PTM) wurde in den 1970er Jahren an der University of Missouri (Kansas City, USA) und an der Maastricht University (Niederlande) unabhängig voneinander entwickelt [24, 25]. Der Maastrichter Progress Test war Vorbild für die Entwicklung des Progress Test Medizin an der Charité - Universitätsmedizin Berlin, der dort 1999 eingeführt wurde. 2000 startete die Charité eine Kooperation mit weiteren medizinischen Universitäten in Deutschland und Österreich [26]. Seit 2010 wird dieser an der MedUni Wien eingesetzt.
Der interdisziplinäre PTM deckt jenes Wissensniveau $\mathrm{ab}$, welches Studierende am Ende ihres Medizinstudiums haben sollen. Er dient primär der Ermittlung des Wissensfortschrittes. Von Jahr zu Jahr können die Studierenden mehr Fragen beantworten, wodurch sich ihr "progress“ stetig erhöht. Der PTM, bestehend aus 200 Prüfungsfragen, wird im MultipleChoice-One-Best-Answer-Fragen-Format mit bis zu sechs Antwortmöglichkeiten durchgeführt. Die Prüfungszusammenstellung erfolgt an der Charité entsprechend einem Blueprint. Der Testwert setzt sich aus der Anzahl an richtigen minus falschen Antworten zusammen. Diese Zahlen werden zum einen mit dem Mittelwert der eigenen Studierendenkohorte und zum anderen dem Mittelwert aller Studierendengruppen und bisherigen TeilnehmerInnen der miteinander kooperierenden österreichischen und deutschen Universitäten aus demselben Semester gegenübergestellt. Als Feedback erhalten die Studierenden Rückmeldung über die Performanz ihrer Teilnahmen, aufgeschlüsselt nach Fachgebieten und Organsystemen. Der PTM hat eine sehr hohe Reliabilität. Ein Hinweis auf die Gültigkeit ist der kontinuierliche Anstieg von richtigen Antworten im Laufe der Jahre [24].

\section{e-PTM}

Um den Progress Test Medizin ökonomischer zu gestalten, wurde dieser im Studienjahr 2017/18 erstmals in elektronischer Form unter Nutzung der Lernplattform Moodle umgesetzt. Damit erhielten die Studierenden die Möglichkeit diesen Test ortsunabhängig zu absolvieren. Die Studierenden mussten sich für die Prüfung - wie gewohnt - anmelden sowie ein Zeitfenster von $3 \mathrm{~h}$ für die Bearbeitung des PTM auswählen. Ersten Rückmeldungen zufolge erhöhte die Umstellung von einer SIP-ähnlichen Papier-Bleistift-Prüfung zu einer elektronischen Prüfung die Akzeptanz der Studierenden.

\section{Continuous Assessment als besondere Heraus- forderung und als Möglichkeit für eine bessere Lernsteuerung der Studierenden}

Studien zeigen, dass wiederholtes Prüfen zu einem höheren Lernzuwachs führt als ressourcenintensive Lehrformate [27]. Es stellt sich daher die Herausforderung, geeignete Methoden zur kontinuierlichen Beurteilung des Lernfortschritts im Studium zu entwickeln und entsprechend anzuwenden. So sollten neben den schriftlichen summativen integrierten Prüfungen (SIP) vermehrt mündliche und praktische Prüfungsformen eingesetzt werden. Innerhalb eines Continuous Assessment Systems muss zudem sichergestellt sein, dass in regelmäßigen Abständen möglichst oft formatives Feedback erfolgt und den Studierenden die Kriterien für ein erfolgreiches Bestehen von Prüfungen klar ist [28]. Daher wird es notwendig sein, vermehrt formative Assessmentele- 
mente als didaktische Reflexion in den Unterricht einzubauen und möglichst zeitnah den Studierenden Rückmeldung über ihren Leistungsstand zu geben. Die Lernsteuerung der Studierenden sollte dahingehend optimiert werden, dass die Prüfungsformen motivieren, schon während des Studienjahres laufend mitzulernen, um damit das „Bulimie-Lernen“ am Ende des Semesters bzw. Studienjahres für die SIP zu reduzieren.

\section{Ausblick}

Ein wesentliches Ziel der MedUni Wien ist die Intensivierung der Leistungsbeurteilung in den Lehrveranstaltungen mit prüfungsimmanentem Charakter. Dazu sollen mündliche und praktische Prüfungsformate ausgebaut und weiterentwickelt werden. Dies erfordert ein Zusammenwirken von Beurteilungsformaten, die das kontinuierliche Lernen unterstützen, d.h. formatives Assessment mit Feedback in Kombination mit summativen Prüfungen, die Entscheidungscharakter haben. Ein weiteres Ziel ist die Ausweitung der Return-Week zu einer abschließenden summativen Prüfung. Damit soll entsprechend der Empfehlungen des Advisory Boards der MedUni Wien eine international vergleichbare Abschlussprüfung für das Studium der Humanmedizin etabliert werden. Orientierungspunkte für die Vergleichbarkeit des Outcomes bilden dabei die Eidgenössische Prüfung in Humanmedizin [29] sowie der deutsche Masterplan Medizinstudium 2020 [30].

Funding Open access funding provided by Medical University of Vienna.

Interessenkonflikt M. Himmelbauer, D. Koller, A. Bäwert und W. Horn geben an, dass kein Interessenkonflikt besteht.

Open Access Dieser Artikel wird unter der Creative Commons Namensnennung 4.0 International Lizenz (http:// creativecommons.org/licenses/by/4.0/deed.de) veröffentlicht, welche die Nutzung, Vervielfältigung, Bearbeitung, Verbreitung und Wiedergabe in jeglichem Medium und Format erlaubt, sofern Sie den/die ursprünglichen Autor(en) und die Quelle ordnungsgemäß nennen, einen Link zur Creative Commons Lizenz beifügen und angeben, ob Änderungen vorgenommen wurden.

\section{Literatur}

1. Scouller K. The influence of assessment method on students' approaches: multiple choice question examination versus assignment essay. High Educ. 1998;35:453-72.

2. TangKCC.Perceptions oftaskdemand, strategyattributions and student learning. Res Dev High Educ. 1992;15:474-81.

3. Seegers M, Gijbels D. The relationship between students' perceptions of portfolio assessment practice and their approaches tolearning. Educ Stud. 2008;34(1):35-44.

4. Wormald BW, Schoeman S, Somasunderam A, Penn M. Assessment drives learning: an unavoidable truth? Anat Sci Educ. 2009;2(5):199-204. https://doi.org/10.1002/ase.102.
5. Wood T. Assessment not only drives learning, it may also helplearning. MedEduc. 2009;43:5-6.

6. Fischer MR, Holzer M, Jünger J. Prüfungen an den medizinischen Fakultäten-Qualität, Verantwortung und Perspektiven. GMS Z Med Ausbild. 2010;27(5):Doc66. https:// doi. org/10.3205/zma000703.

7. Miller GE. The assessment of clinical skills/competence/ performance. Acad Med. 1990;65:S63-S7.

8. Bennett CR, Mawhood N, Platt MJ. The impact of medical school assessment on preparedness for practice. Med Teach. 2018;18:1-3. https://doi.org/10.1080/0142159X. 2018.142579.

9. Biggs J, Tang C. Teaching for quality learning at university: what the student does. 4. Aufl. Berkshire: McGraw-Hill; 2011.

10. Medizinische Universität Wien. Curriculum Diplomstudium Humanmedizin N202 (kons. Fassung) Studienjahr 2017/18. 2017. https://www.meduniwien.ac.at/web/ fileadmin/content/serviceeinrichtungen/ studienabteilung/studium/Humanmedizin/ StudienplanN202_2017.pdf.Zugegriffen:17.Juli 2018.

11. Bloch R, Hofer D, Krebs R. Kompetent prüfen: Handbuch zur Planung, Durchführung und Auswertung von Facharztprüfungen.Institutfür MedizinischeLehreIML:Universität Bern; 1999.

12. Kopp V, Möltner A, Fischer MR. Key-Feature-Probleme zum Prüfen von prozeduralem Wissen:Ein Praxisleitfaden.GMS ZMed Ausbild. 2006;23(3):1-6.

13. Carraccio C, Englander R. The objective structured clinical examination. A step in the direction of competency-based evaluation. Arch Pediatr Adolesc Med. 2000;154(7):736-41.

14. Palarm TW, Griffiths M, Phillips R. The design, implementation and evaluation of electronic objective structured clinical examinations in diagnostic imaging: an "action research" strategy. J Diagn Radiogr Imaging. 2004;5(2):79-87.

15. Norcini JJ. The mini-clinical evaluation exercise (miniCEX). Clin Teach. 2005;2(1):25-30.

16. Zlabinger G, Anvari-Pirsch A, Horn W, Kainberger F, Steinlechner B, Hrsg. Logbuch. Wien: MedUni; 2017.

17. Zlabinger G, Anvari-Pirsch A, Horn W, Kainberger F, Steinlechner B, Hrsg. Logbuch und Portfolio Klinisch-Praktisches Jahr. Wien: MedUni; 2017. S. vi.

18. Rushton A. Formative assessment: a key to deep learning? Med Teach. 2005;27(6):509-13.

19. Nouns Z, Georg W. Progress testing in German speaking countries. Med Teach. 2010;32:467-70.

20. Schuwirth L, Van der Vleuten C. The use of progress testing. Perspect Med Educ. 2012;1:24-30.

21. Jünger J. Kompetenzorientiert prüfen im Staatsexamen Medizin. Bundesgesundheitsblatt Gesundheitsforschung Gesundheitsschutz. 2017;61(2):171-7. https://doi.org/10. 1007/s00103-017-2668-9.

22. Martinez M, Lipson J. Assessment for learning. Educ Leadersh. 1989;47:73-5.

23. Schuwirth L, Van der Vleuten C. Programmatic assessment: From assessment of learning to assessment for learning. Med Teach. 2011;33(6):478-85.

24. Arnold L, Willioughby T. The quarterly profile examination. Acad Med. 1990;65(8):515-6.

25. Albano M, Cavallo F, Hoogenboom R, Magni F, Majoor G, Mananti F, et al. An international comparison of knowledge levels of medical students: the Maastricht Progress Test. MedEduc. 1996;30(4):239-45.

26. Charité. Progress Test Medizin. 2018. https://ptm.charite. de.Zugegriffen: 19. März2018.

27. Raupach T, Brown J, Anders S, Hasenfuss G, Harendza S. Summative assessments are more powerful drivers of student learning than resource intensive teaching formats. BMCMed.2013;11:61. 
28. Rust C. The impact of assessment on student learning. Act Learn High Educ. 2002;3(2):145-58.

29. Bundesamt für Gesundheit BAG. Eidgenössische Prüfung in Humanmedizin. 2018. https://www.bag.admin.ch/ $\mathrm{bag} / \mathrm{de} / \mathrm{home} /$ themen/berufe-im-gesundheitswesen/ medizinalberufe/eidgenoessische-pruefungenuniversitaerer-medizinalberufe/eidgenoessische- pruefung-in-humanmedizin.html. Zugegriffen: 17. Juli 2018.

30. Bundesministerium für Bildung und Forschung. Masterplan Medizinstudium 2020. 2017. https://www.bmbf.de/ files/2017-03-31_Masterplan\%20Beschlusstext.pdf.Zugegriffen: 17. Juli 2018. 